eISSN: 2659-6482

DOI: https://doi.org/10.14201/pmrt.20824

\title{
EL MAL QUERER (2018) DE ROSALÍA: SEMIÓTICA DEL VIDEOCLIP «DI MI NOMBRE (CAP. VIII: ÉXTASIS)»
}

\author{
Rosalia's El mal querer (2018): «Di mi nombre \\ (Cap. VIII: Éxtasis)» videoclip semiotics
}

\author{
Carlos MORALES GÁLVEZ \\ Universidad de Granada \\ Cmoralesgalvez@correo.ugr.es
}

RESUMEN: A finales de 2018 la cantante Rosalía Vila Tobella (Barcelona, 1993) presenta su segundo álbum, titulado El mal querer, obteniendo gran visibilidad en los medios de comunicación internacionales, además de premios tan significativos como dos Grammys Latinos. Paralelamente, Rosalía como fenómeno musical ha generado una fuerte controversia en torno a la acusación de apropiación cultural, ya incipiente desde comienzos del mismo año a raíz de su anterior trabajo. Debido a ello, han surgido un buen número de vídeos divulgativos en la plataforma YouTube enfocados a la puesta en valor del contenido y dignificación musical del álbum. Sin embargo, aún no se ha planteado un análisis musical exhaustivo de sus canciones, videoclips o la propia campaña publicitaria adoptada por Rosalía. En este sentido, con el presente trabajo se pretende realizar un estudio semiótico y hermenéutico de la canción que consta de un videoclip en su canal oficial de YouTube: «Di mi nombre (Cap. VIII: Éxtasis)». Además, se implementarán propuestas de análisis iconográfico, desde una perspectiva multidisciplinar, y se precisarán cómo los rasgos de los géneros musicales urbanos que se han hibridado con el flamenco actúan en la música de Rosalía. Así pues, se identificarán varios procesos de intertextualidad y se determinará el imaginario simbólico como metodología para demostrar la hipótesis de trabajo.

Palabras clave: Rosalía; semiótica; intertextualidad; flamenco; hibridación musical. 
ABSTRACT: At the end of 2018 the singer Rosalía Vila Tobella (Barcelona, 1993) released her second album, entitled El mal querer, acquiring great visibility in the international media, as well as significant awards such as two Latin Grammys. Simultaneously, as a musical phenomenon she has generated strong controversy on the cultural appropriation accusation, yet emerging since the beginning of the same year due to her previous work. In consequence, there has arisen a considerable number of educational videos in the YouTube platform focused on promoting the album content and musical value by dignifying it. However, there has not been an exhaustive musical analysis proposal of its songs, video clips or the marketing campaign itself adopted by Rosalía. In this sense, it is intended in this article to carry out a semiotic and hermeneutic study of the song that also has a video clip in her official YouTube channel: «Di mi nombre (Cap. VIII: Éxtasis)». In addition, iconographic analysis methodologies would be implemented, with a multidisciplinary perspective, and how the characteristics of the urban musical genres hybridized with flamenco interact in Rosalía's music with each other. Thus, several intertextuality processes would be identified and the symbolic imaginary would be specified as a method to prove the work hypothesis.

Key words: Rosalía; semiotics; intertextuality; flamenco; musical hybridization.

\section{INTRODUCCIÓN}

El presente trabajo versa sobre el último álbum de Rosalía Vila Tobella (2018a) -una artista emergente nacida en Barcelona en 1993-, el cual conforma su Proyecto Final de Carrera en la Escuela Superior de Música de Cataluña para la obtención del Título Superior (Gallardo, 2018) y con el que ha obtenido cinco nominaciones a los Grammys Latinos en la edición de 2018 y ganado dos (AA. VV.).

Como hipótesis de trabajo he considerado que la fusión del imaginario español de Rosalía con elementos de diversos géneros musicales urbanos ha dado lugar a una hibridación de repercusión internacional en tanto a la susceptibilidad de dichos elementos para ser reconocidos por el público. Este argumento se sustenta a través de que, si el público está previamente familiarizado con la idea que se intenta transmitir en un producto, entonces existe una mayor probabilidad de consumirlo (Auty y Lewis, 2004: 117-133).

Así pues, los objetivos de esta investigación han sido determinar el imaginario simbólico incluido en la obra de Rosalía en su interrelación con los componentes 
musicales y culturales; precisar cómo se han hibridado los géneros musicales urbanos y el flamenco en la música de Rosalía; identificar los procesos de intertextualidad sonora y visual en el objeto de estudio.

Para ello, he realizado un análisis del videoclip partiendo de un estudio semiótico e implementando propuestas de análisis iconográfico, en conjunción con los elementos sonoros desde una perspectiva multidisciplinar. En concreto, dicho objeto de estudio es la canción que consta de videoclip en su canal oficial de YouTube y que recibe por título «Di mi nombre (Cap. VIII: Éxtasis)» (Vila Tobella, 2018b).

\section{EL MAL QUERER (2018): CONTEXTUALIZACIÓN}

\subsection{Estructura}

Estudiar los elementos generales del disco y la estética global aplicada a la música permitirá comprender mejor el análisis propuesto. La estructura se organiza en once pistas siguiendo un modelo narrativo en el que cada canción cuenta un capítulo de la historia; se trata, por tanto, de un álbum conceptual. Debido a que consta de una duración total de 30 minutos y 14 segundos, el disco se adecúa a los tiempos de consumo de la sociedad actual y permite su audición de principio a fin sin demasiada dificultad.

\begin{tabular}{|c|c|}
\hline \multicolumn{2}{|c|}{ Tabla 1. Estructura del disco } \\
\hline Título & Duración \\
\hline «Malamente (Cap. I: Augurio)» & {$[02: 30]$} \\
\hline «Que no salga la luna (Cap. II: Boda)» & {$[04: 29]$} \\
\hline «Pienso en tu mirá (Cap. III: Celos)» & {$[03: 13]$} \\
\hline «De aquí no sales (Cap. IV: Disputa)» & {$[02: 24]$} \\
\hline «Reniego (Cap. V: Lamento)» & {$[03: 28]$} \\
\hline «Preso (Cap. VI: Clausura)» & {$[00: 40]$} \\
\hline «Bagdad (Cap. VII: Liturgia)» & {$[03: 02]$} \\
\hline «Di mi nombre (Cap. VIII: Éxtasis)» & {$[02: 42]$} \\
\hline «Nana (Cap. IX: Concepción)» & {$[03: 17]$} \\
\hline
\end{tabular}


CARLOS MORALES GÁLVEZ

EL MAL QUERER (2018) DE ROSALÍA: SEMIÓTICA DEL VIDEOCLIP «DI MI NOMBRE (CAP. VIII: ÉXTASIS)»

\begin{tabular}{|c|c|}
\hline \multicolumn{2}{|c|}{ Tabla 1. Estructura del disco } \\
\hline Título & Duración \\
\hline «Maldición (Cap. X: Cordura)» & {$[02: 55]$} \\
\hline «A ningún hombre (Cap. XI: Poder)» & {$[01: 34]$} \\
\hline
\end{tabular}

La trama comienza revelando, en primera persona, el destino trágico al que será sometida la protagonista. A continuación, se narra, desde el punto de vista del novio, la boda; la tercera y cuarta canción también se sirven de este punto de vista y se basan en los celos crecientes que sufre el marido, las disputas y el maltrato físico. El quinto lugar sirve para volver al punto de vista narrativo de la chica y consiste en una manifestación de su resignación en público y tristeza interior. La sexta pista podría considerarse una sección de transición situada en un punto futuro y muy distante de la historia global del disco, en primera persona, o en el mismo decurso de la narración, pero con una narradora distinta de la protagonista. Su argumento alude a un matrimonio terrible en el cual los hijos suponen la redención del mismo. Seguidamente, un narrador omnisciente introduce a la mujer atrapada y secuestrada por su marido. La octava canción habla, en primera persona -al igual que el resto del álbum-, de cómo la mujer es sometida a los deseos sexuales del cónyuge; se produce la concepción. Metafóricamente, la nana que le sigue revela que el hijo, fruto del matrimonio, ha nacido muerto. La penúltima pista descubre el desenlace del nudo: ella asesina al marido como vía para obtener la liberación. Por último, ese acto conllevará unas consecuencias a las que habrá de enfrentarse, aunque el último track afirma que no dejará que ningún hombre dicte sentencia alguna.

\subsection{Géneros musicales: flamenco y pop}

En cuanto a los géneros musicales, el flamenco supone tanto el punto de partida como la base de todo el trabajo. La utilización de rasgos estéticos pertenecientes a palos flamencos como las bulerías [Track n. ${ }^{\circ} 2$ ], las seguiriyas [Track n. ${ }^{\circ}$ 5], tintes de soleá [Track n. ${ }^{\circ} 6$ ], tangos [Track n. ${ }^{\circ} 8$ ], una nana [Track n. ${ }^{\circ} 9$ ] y un cierto cante tipo fandango [Track n. ${ }^{\circ} 10$ ], con préstamos de diversos autores y la tradición folklórica, se incluyen durante la grabación; así, contienen métricas como la cuarteta octosilábica con alteraciones [Tracks n. ${ }^{\circ}$ 1, 7, 8, 9, 11]; la quintilla octosilábica con la repetición del segundo verso antes de su comienzo [Track n. ${ }^{\circ} 10$ ]; la métrica de la seguiriya, es decir: 6/6/11/6, pero con repeticiones y variaciones [Track . $^{\circ}$ 5], y su típica entrada: "Tirí, tirí, tirí, yayay». En lo relativo al compás, aparece el típico compás binario de tango [Tracks n. ${ }^{\circ} 1,7,8$ ], el compás de amalgama de 
doce unidades [Tracks $\left.n .^{\circ} 2,3,4,5,6\right]$ y una percusión con chasquidos abandolaos [Track n. $\left.{ }^{\circ} 10\right]$. La voz posee atributos que oscilan entre la claridad y la oscuridad debido a la dicción y a la inserción de efectos sonoros; cabe destacar el uso de palabras en idioma caló como «Undebel», «sacais», «camelo», «ducas» o «duquelas» (Gamella et al., 2012) [Tracks n. ${ }^{\circ} 1,2,4,5$, respectivamente]. Igualmente, se atisban algunas cadencias andaluzas [Tracks $n .^{\circ} 2$ y 8 ] y el jaleo típico manifestado en los coros y las palmas [Track n. ${ }^{\circ}$ ].

Aquí radica parte del éxito y repercusión que tendrá este álbum en el futuro -y que ya está teniendo-, es decir, la combinación de cualidades asociadas con el andalucismo tiene un peso específico ya que, a lo largo de los últimos siglos, ha formado parte del imaginario internacional y la forma de percibir la España musical en el extranjero (Giménez Rodríguez, 2008: 160). Y esto gesta un lenguaje inteligible y accesible a la audiencia mundial.

Por otro lado, lo urbano y determinados elementos de música electrónica reflejan una llamativa sinergia al hibridarse con el flamenco. Algunas palabras de fondo-ad-libs-pertenecientes a un vocabulario coloquial como «illo»o «trá trá» se manifiestan en las pistas; efectos de sirenas, coches, motos e, inclusive, espadas atravesando cuerpos son introducidos para intensificar la atmósfera urbana que se genera -la pista n. ${ }^{\circ} 4$ explota estos recursos rememorando a Björk en un proceso intertextual-. En lo referente a la música electrónica, más que como género, se hace uso extensivo de recursos como una línea de bajo extrema y potente -subgraves-; una acentuación en las partes fuertes de cada pulso o de cada compás; la incorporación de sampleos sencillos y cortos -con ruedas de unos pocos acordes realizados por un sintetizador-, y efectos y filtros en la voz como el voice harmonizer o reverb. Como es de esperar, todas estas características no se usan como mero fin, sino como medio para apoyar la narración de la historia, desarrollar un concepto e hibridar tópicos musicales.

Por tanto, diría que los rasgos de las músicas urbana y electrónica se pueden englobar en un conjunto mayor al que denominaré pop. En este sentido, como apelación y reivindicación de esta categorización existe otro proceso intertextual insertado en la pista . $^{\circ} 7$ y en su videoclip (Vila Tobella, 2018c). Se trata del préstamo de la melodía de una canción de Justin Timberlake (Memphis, 1981): «Cry Me a River», el cual provoca un reconocimiento e identificación con el producto musical por parte de la audiencia internacional al provenir de una canción tan reproducida en el Billboard Hot 100 -alcanzó el puesto n. 3 en 2003- (Timberlake, 1981-2019) y cuyo álbum (Justified) llegó a vender más de 10 millones de copias. Pero, por si fuera poco, el tema se inspiró en la ruptura del propio J. Timberlake con Britney Spears (Misisipi, 1981), situación paralela a la ruptura de Rosalía con C. Tangana (Antón Álvarez Alfaro, Madrid, 1990) en 2018. Así pues, el hecho de que Rosalía vaya vestida con un mono rojo similar al famoso 
red catsuit de Britney Spears en «Oops I Did It Again» (1999), conociendo el trasfondo personal, alude a otra intertextualidad que tiene como objetivo asentar el universo e imaginario pop en el consumidor ya que no hay que olvidar que los géneros musicales también se definen a través de reglas sociales e ideológicas que no tienen por qué ser musicales (Fabbri, 1981: 53-59). Por último, cabe mencionar que las dos canciones citadas fueron desarrolladas para la misma discográfica a la que pertenecían sus cantantes: Jive Records. Esta es la casa a la que actualmente pertenece a Sony Music: el sello de El mal querer.

\section{ANÁLISIS DEL VIDEOCLIP: «DI MI NOMBRE (CAP. VIII: ÉXTASIS)»}

\subsection{Autorias}

Como primer punto, la elaboración visual del objeto de estudio corre a cargo de Caviar, productora que ya aglutina dos videoclips de Dua Lipa (Londres, 1995): «New Rules» (2017) y «IDGAF» (2018). Ambos han sido dirigidos por Henry Scholfield; es quien, curiosamente, ha adquirido el mismo rol en «Di mi nombre (Cap. VIII: Éxtasis)» (Caviar, 2010-2019). Este detalle es significativo en tanto que Dua Lipa llegó a ostentar el sexto puesto en el Billboard de 2018 con la canción «New Rules» (Lipa, 1995-2019) y recibir 1.723 millones de visitas en el vídeo subido a YouTube (Lipa, 2017). Por tanto, «Di mi nombre (Cap. VIII: Éxtasis)» se sustenta mediante una estrategia de marketing verdaderamente compleja englobada dentro de un proceso -entre varios- aún mayor: la publicación periódica y esparcida de los videoclips puesto que primero fue «Malamente (Cap. I: Augurio)» el 29 de mayo de 2018 (Vila Tobella, 2018d); después le siguió «Pienso en tu mirá (Cap. III: celos)» el 23 de julio de 2018e (Villa Tobella), y a continuación, el 30 de noviembre de 2018, tres días antes de sacar el disco a la venta, salió a la luz el videoclip en cuestión. El objetivo de esta táctica de no es otro que generar expectación entre el público al fragmentar un disco conceptual que narra, generalmente, una historia de manera lineal. Además, hay que tener en cuenta el potencial de los videoclips como medio publicitario en la Red, pero también como generadores de identidad entre los espectadores y canal que permite atribuir y construir significados acerca del artista (Viñuela Suárez, 2013: 172, 183-184).

La autoría de la letra y música corresponde a Antón Álvarez y Rosalía; sobre la música, se reconoce la composición a Rosalía y El Guincho junto con el uso de elementos de dominio público; los intérpretes son Rosalía -voz, coros, ad-libs, palmas, bajo y arreglos-, El Guincho -sintetizador, 808, bajo, ad-libs y arreglos-, Los Mellis -palmas y coros-, Las Negris -coros y jaleo- y Laura Boschetti -arpa-(Vila Tobella, 2018). Asimismo, la coreografía ha sido realizada por Charm La'Donna (California, 1989), bailarina que ha trabajado con Madonna (Míchigan, 1958), Britney Spears o Selena Gómez (Texas, 1992) (La’Donna, 1989-2019). Este 
reconocimiento sobre las diversas autorías en el libreto y en el vídeo, especialmente las de personalidades tan influyentes en el panorama de las músicas populares urbanas como C. Tangana, Charm La'Donna o Henry Scholfield, revela la intención de dirigirse a un target muy concreto: las audiencias de dichos artistas (Viñuela Suárez, 2013: 172). Ello logra la captación de otros sectores que se añaden al público anteriormente existente del que gozaba Rosalía.

\subsection{Letra y música}

Volviendo a recurrir a la definición de género musical, y sus características, acerca de Franco Fabbri (1981: 52-59), será necesaria, aunque no suficiente, la descripción de las estructuras formales y los elementos musicales para identificar y ejemplificar la hibridación planteada en 2.2. Además, dado que la forma viene determinada, en gran medida, por la letra, consideraré lo que en la semiótica se denomina clases de paradigmas -paradigm clases- para asociar eventos dentro de un medio determinado de acuerdo a un número de categorías distintas (Cook, 1999: 142).

\begin{tabular}{|c|c|c|c|}
\hline & Estribillo 1/INTRO & & Estrofa $4=E 4$ \\
\hline \multirow[t]{2}{*}{$\begin{array}{l}e 1 \\
e 2\end{array}$} & $\begin{array}{l}\text { Yali, yali, yali, yali, yali, yali, } \\
\text { yali, ya } \\
\text { li, yali, yali, yali, yali, yali, yali, } \\
\text { ya }\end{array}$ & \multirow[t]{2}{*}{$\begin{array}{l}v 9 \\
v 10 \\
v 11 \\
v 12\end{array}$} & $\begin{array}{l}\text { Di mi nombre (va) } \\
\text { Pon tu cuerpo contra el mío } \\
\text { Y haz que lo malo sea bueno } \\
\text { Impuro lo bendecido (¡ole!) }\end{array}$ \\
\hline & Estrofa $1=E 1$ & & Estrofa $5 /$ PreEstribillo $=E s$ \\
\hline $\begin{array}{l}v 1 \\
v 2 \\
v 2^{\prime} \\
v 2^{\prime}\end{array}$ & $\begin{array}{l}\text { Di mi nombre (eh) } \\
\text { Cuando no haya nadie cerca } \\
\text { Cuando no haya nadie cerca } \\
\text { Cuando no haya nadie cerca } \\
\text { (¡cerca!) (¡ole!) }\end{array}$ & \multirow[t]{3}{*}{$\begin{array}{l}v 13 \\
v 14 \\
v 15 \\
v 16 \\
v 15 \\
v 16\end{array}$} & \multirow{3}{*}{$\begin{array}{l}\text { Y hazme rezar sobre tu cuerpo } \\
\text { En la esquina de tu cama } \\
\text { Y en el último momento } \\
\text { Dime mi nombre a la cara (atrás, } \\
\text { atrás) } \\
\text { Y en el último momento } \\
\text { Dime mi nombre a la cara (atrás, } \\
\text { atrás) } \\
\text { (¡ole!) (tratrán, tratrán, triste, } \\
\text { triste, triste) }\end{array}$} \\
\hline & Estrofa $2=E 2$ & & \\
\hline $\begin{array}{l}v 3 \\
v 3 \\
v 3 ” \\
v 4\end{array}$ & $\begin{array}{l}\text { Que las cosas (seh) } \\
\text { Que las cosas que me dices } \\
\text { Que las cosas que me dices } \\
\text { No salgan por esa puerta (¡ole!) }\end{array}$ & & \\
\hline
\end{tabular}




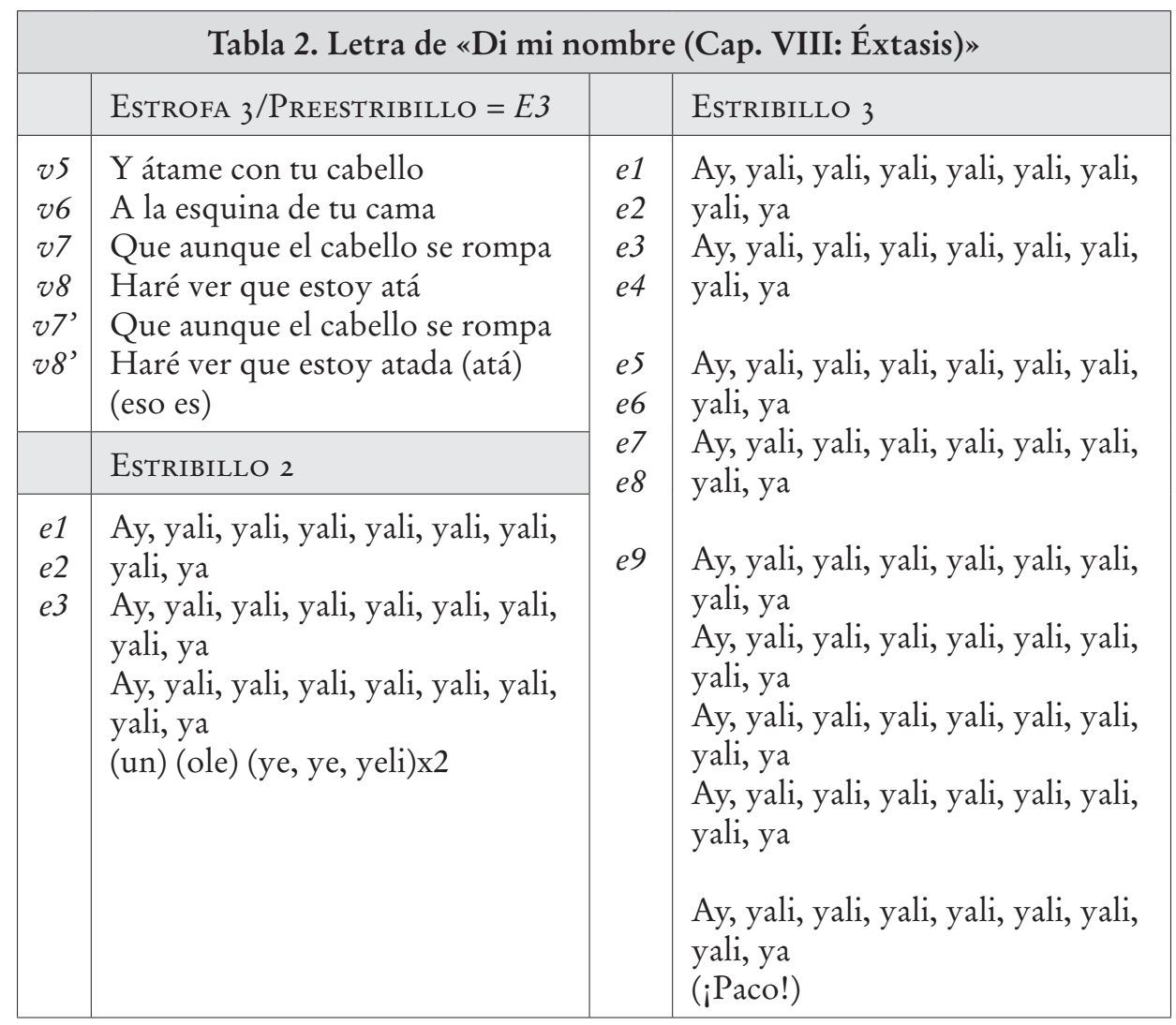

Así pues, la letra de las estrofas se construye sobre una cuarteta octosilábica, la forma métrica más presente en el flamenco junto con la quintilla de seis tercios, pero con las irregularidades propias del género ya que $v 1, v 3, v 8, v 9$ y v13, un verso por cada estrofa, no mantienen la medida de ocho sílabas. Otro rasgo de las métricas en el flamenco es la repetición de versos en las estrofas sin que modifiquen la idea de la cuarteta a la hora de la medida formal. Ello se manifiesta tanto en E1 como en $E 2$, ya que pueden agruparse en una única estrofa, tal y como sucede en el libreto del propio disco; $E 3$ y $E 5$ también son cuartetas porque repiten sus dos últimos versos al final de la misma. La fonética también rememora la dicción oscura y habla andaluza, empleada en el género, transformando la $c$ y la $z$ en la $s$ (cerca, dices, bendecido, rezar $\rightarrow$ /'ser ka/, /'di $\theta$ es/, /'beN de 'si o/, /ře 'sar/), algunas $l$ en $r$ (el, último, salgan $\rightarrow / \mathrm{er} /$, /'ur ti mo/, / 'sar $\gamma$ an /); perdiendo la $s$ (haz, esquina, atrás $\rightarrow /$ ah/, / eh 'ki na/, /a 'trah/) u omitiendo la última consonante en algunas palabras (bendecido, atada $\rightarrow /$ 'beN de 'si o/, /a 'ta/). En este sentido, 
estos elementos contribuyen a generar una función estética coherente con el estilo musical y con la trama de la historia, cuya protagonista es una gitana. En otras palabras: crean un marco verosímil.

Sin embargo, el aspecto más interesante lo aporta la palabra yali, o yeli, porque no se distingue bien si la pronunciación es abierta o semiabierta, y porque en el flamenco no suele hacerse uso de los estribillos; se trata de un detalle clave que aporta dos niveles de intertextualidad: rememora los Tangos de Málaga de Enriqueta Reyes Porras, conocida como la Repompa de Málaga (Málaga, 1937-Málaga, 1959), o los tangos granadinos, y el ritual de boda gitana conocido como «el Yeli». Realmente, dentro de la narración de esta canción, supone un momento en el que se alude a la boda que acontece en el track n. ${ }^{\circ} 2$ "Que no salga la luna (Cap. II: Boda)»: es una autorreferencia. Idea que se refuerza con la metáfora del $v 7-$ la pérdida de la virginidad-y v10, v11 y v12 -el acto de mancillar algo virgen-. Además, dicha palabra articula la estructura musical dando lugar al Estribillo.

\begin{tabular}{|c|c|c|c|c|c|c|c|c|}
\hline \multicolumn{9}{|c|}{ Tabla 3. Estructura formal de la música } \\
\hline $\begin{array}{c}\text { ESTRIBILLO } \\
1 \text { INTRO }\end{array}$ & E1 & E2 & E3 & $\begin{array}{c}\text { ESTRIBILLO } \\
2\end{array}$ & $E 4$ & ES & $\begin{array}{c}\text { ESTRIBILLO } \\
3\end{array}$ & $\begin{array}{l}\text { Plano y } \\
\text { CRÉDITOS }\end{array}$ \\
\hline$[0: 02-0: 12]$ & {$[0: 12]$} & {$[0: 25]$} & {$[0: 38]$} & {$[0: 59]$} & {$[1: 14]$} & {$[1: 33]$} & [1:54] & {$[2: 42-3: 00]$} \\
\hline
\end{tabular}

Por otra parte, la melodía se desarrolla en un modo de mi sobre re\#, es decir, modo frigio en re\#, pero con la tercera alterada y la presencia de cuartos de tono puntuales en los adornos. Son dos elementos muy característicos de las músicas populares y, en concreto, del flamenco. En cuanto al fraseo, este se construye sobre los tercios, de manera anacrúsica y asincopada, con semifrases de 2 y $4 \mathrm{cc}$.: $E 1$ (4cc.) funciona de antecedente de $E 2$ ( $4 \mathrm{cc}$.), que actúa como su consecuente terminando sobre una cadencia andaluza; $E 3(2 \mathrm{cc} .+4 \mathrm{cc}$.) contiene el mismo material que E5, ya que son los Preestribillos, y conducen hacia el EstribiLLO 2 y 3 generando la tensión en los dos primeros compases y resolviéndola en los cuatro siguientes, de esta forma se mantiene la resolución en la tónica al final de cada semifrase en todas las estrofas; $E 4$ equivale a E2; el Estribillo 1 consta de 4cc. y no presenta variaciones en su fraseo; el Estribillo 2 (2cc. + $2 \mathrm{cc} .+2 \mathrm{cc}$.) resuelve con la primera y la tercera semifrase en re\#, pero alterna en la segunda con el fa\# para provocar tensión y variedad; de igual manera ocurre con el Estribillo 3, que alterna las nueve semifrases resolviendo en re\# o en fa\#. Todo ello apunta a otros rasgos del flamenco: la insistencia en la tónica y el fraseo ceñido al cante de los tercios. 

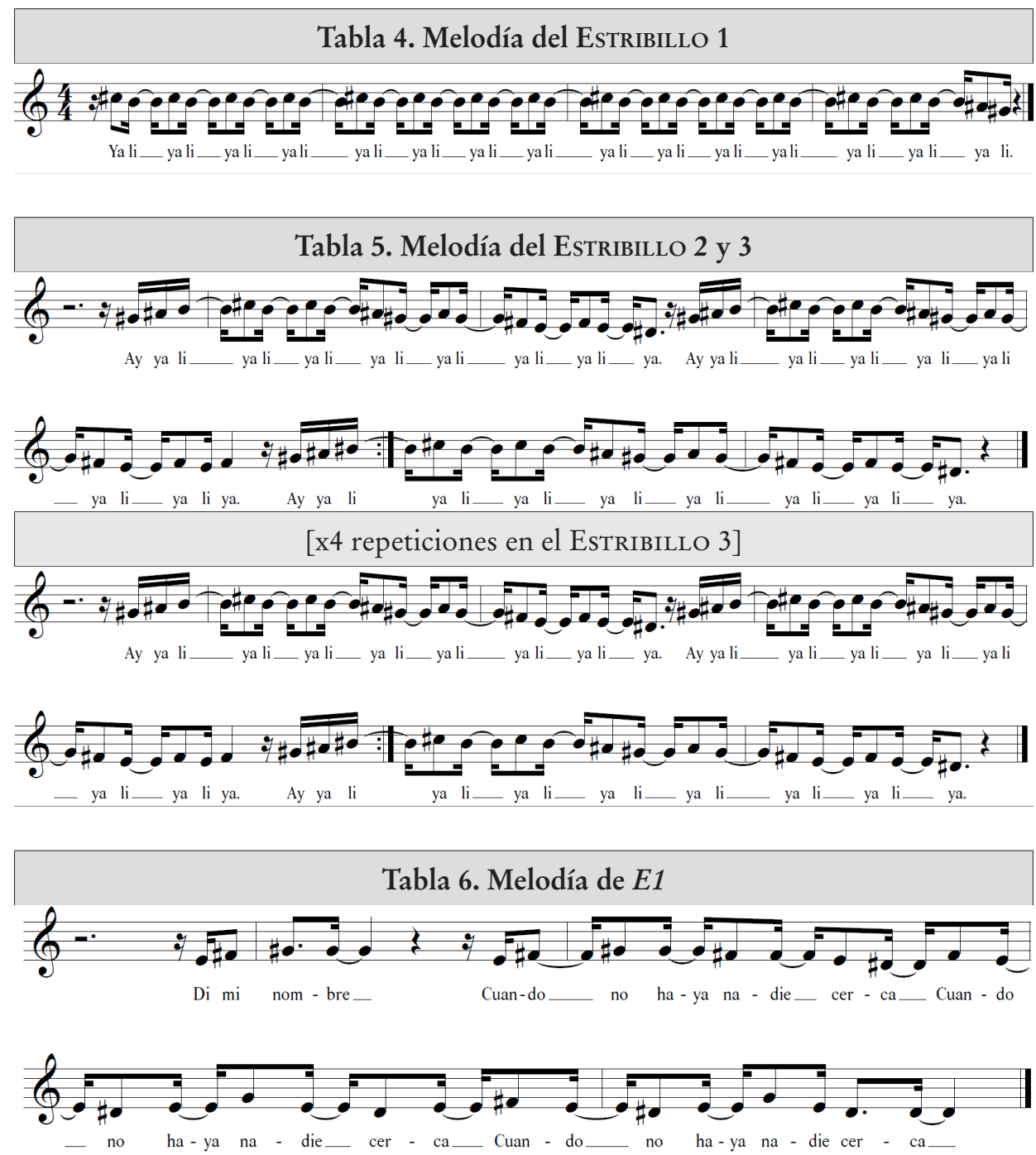

Ediciones Universidad de Salamanca / ®@ PopularMusicResearchToday,2,1(2020),pp.5-23 

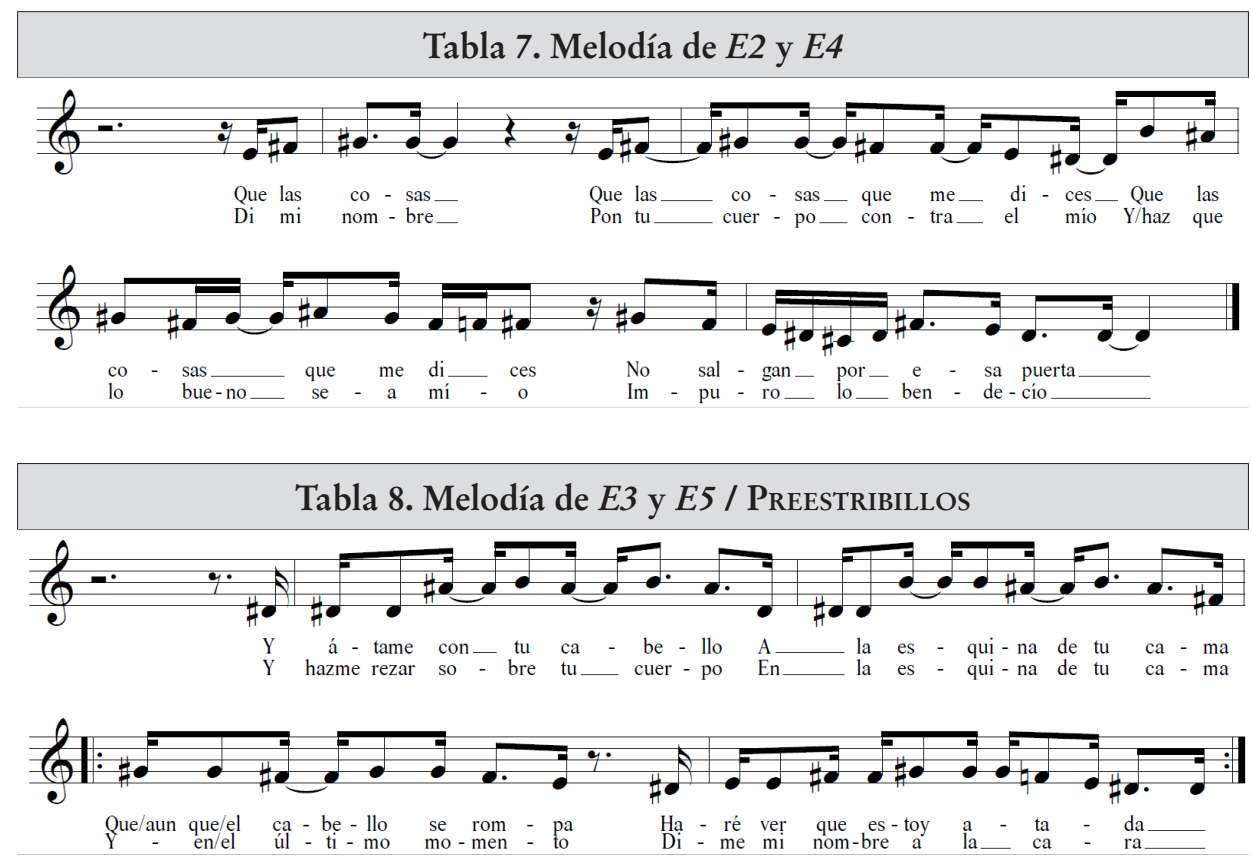

Asimismo, las intervenciones que se realizan en ad-libs al final de los versos $v 1, v 3, v 9, v 9^{\prime}, v 16$ y $v 16^{\prime}$ se entrelazan con la introducción de un jaleo de fondo patente al final de los versos de cada estrofa y en los Estribillos 1 y 2, así como desde $e 3$ a $e 9$ en el Estribillo 3. Por tanto, se ha hibridado un código de las músicas urbanas con otro del flamenco, ambos con cierto componente de ritual en sus correspondientes contextos.

Los efectos añadidos a la voz también cumplen un papel específico directamente relacionado con la tímbrica y desarrollado en el siguiente epígrafe. En el plano sonoro de la letra realizan una función de subrayado musical y una función estética, es decir, resaltan su significado e introducen una característica muy frecuente en la música pop y urbana; aunque bien podrían relacionarse con un cambio en la personalidad del personaje ya que otorgan un carácter robótico, como si estuviera alienada. A continuación, se utiliza la $\mathrm{H}$ como abreviatura para el harmonizer; la flecha hacia arriba $(\uparrow)$ indica la superposición de una $3 .^{a}$ o $5 .^{a}$ por encima y la flecha hacia abajo $(\downarrow)$ en sentido contrario.

El Estribillo i solo incluye AutoTune, pero el Estribillo 2 y el EstribiLLo 3 recurren tanto al AutoTune como al harmonizer, en el segundo se aplica puntualmente en $e 2$ y $e 3$; en el tercero se aplica desde los versos $e 2$ a $e 8$ siguiendo la misma lógica que en el Estribillo 2. 


\begin{tabular}{|l|l|l|}
\hline \multicolumn{3}{|c|}{ Tabla 9. Harmonizer en los Estribillos } \\
\hline Estribillo 2 & $e 2-e 3$ & $\begin{array}{l}\text { Ay, yali, ya }[\mathrm{H} \uparrow] \text { li, yali, ya }[\mathrm{H} \uparrow] \text { li, yali, ya }[\mathrm{H} \uparrow] \text { li, yali, } \\
\text { ya }\end{array}$ \\
\hline Estribillo 3 & $e 2-e 8$ & $\begin{array}{l}\text { Ay, yali, ya }[\mathrm{H} \uparrow] \text { li, yali, ya }[\mathrm{H} \uparrow] \text { li, yali, ya }[\mathrm{H} \uparrow] \text { li, yali, } \\
\text { ya }\end{array}$ \\
\hline
\end{tabular}

En las estrofas solo se emplea el harmonizer en determinadas palabras como «nombre» en $v 1(\mathrm{H} \uparrow)$, «icerca!» en $v 2(\mathrm{H} \uparrow)$, «que» en $v 3(\mathrm{H} \uparrow)$, «no» en $v 4$ $(\mathrm{H} \downarrow)$, «nombre» en $v 9(\mathrm{H} \uparrow)$, «haz» en $v 11$ e «impuro» en $v 12(\mathrm{H} \downarrow)$; partes de versos como «con tu cabello» en $v 5(\mathrm{H} \uparrow)$; todo $v 6(\mathrm{H} \uparrow), v 7(\mathrm{H} \downarrow), v 8(\mathrm{H} \downarrow), v 7^{\text {' }}$ $(\mathrm{H} \uparrow+\mathrm{H} \downarrow)$ y $v 8^{\prime} \mathrm{H} \downarrow$. De manera análoga sucede en E5: v13 $(\mathrm{H} \uparrow), v 14(\mathrm{H} \uparrow), v 15$ $(\mathrm{H} \downarrow), v 16(\mathrm{H} \downarrow), v 15^{\prime}(\mathrm{H} \uparrow+\mathrm{H} \downarrow)$ y $v 16^{\prime}$ sin efectos.

\subsection{Música, imagen y coreografía}

En cuanto al acompañamiento armónico, destaca el gran número de cadencias andaluzas: diez perfectas y dos que no resuelven en la tónica sino en fa\# mayor -o un re\#7 sin la fundamental-. Y, por otra parte, dado el uso del II grado (mi), como acorde de dominante que resuelve en la tónica I (re\#), entonces la función estructural de esta armonía es modal.

\begin{tabular}{|l|l|}
\hline \multicolumn{2}{|c|}{ Tabla 10. Armonía de las Estrofas } \\
\hline E1 & $*$ mi re\# | mi re\# | mi re\# $\mid$ \\
\hline$E 2 / E 4$ & $*$ mi $\mid$ mi re\# | sol\#m fa\# $\mid$ mi re\#| \\
\hline$E 3 / E 5$ & $*|-|-\mid$ sol\#m fa\# | mi re\#: $\mid$ \\
\hline
\end{tabular}

\begin{tabular}{|c|c|c|c|}
\hline \multicolumn{4}{|c|}{ Tabla 11. Armonía de los Estribillos } \\
\hline $\begin{array}{l}\text { Estribillo } \\
1\end{array}$ & $\begin{array}{l}e 1|\mathrm{sol} \# \mathrm{~m}| \\
\text { sol\#m } \\
e 2|\mathrm{re} \# 7| \mathrm{re} \# 7 \mid\end{array}$ & $\begin{array}{l}\text { EstRIBILlo } \\
2\end{array}$ & $\begin{array}{l}e 1 * \mid \text { sol\#m }|\mathrm{fa} \#| \mathrm{mi}|\mathrm{re}| \\
e 2 * \mid \text { sol\#m } \mid \mathrm{fa} \text { | mi }|\mathrm{fa} \#| \\
e 3 * \mid \text { sol\#m }|\mathrm{fa} \#| \mathrm{mi}|\mathrm{re}|\end{array}$ \\
\hline $\begin{array}{l}\text { EstRIBILLO } \\
3\end{array}$ & \multicolumn{2}{|c|}{$\begin{array}{l}e 1 / e 3 * \mid \text { sol\#m } \mid \text { fa\# }|\mathrm{mi}| \\
\text { re\#| } \\
e 2 / e 4 * \mid \text { sol\#m } \mid \text { fa\# }|\mathrm{mi}| \\
\mathrm{fa} \mid\end{array}$} & 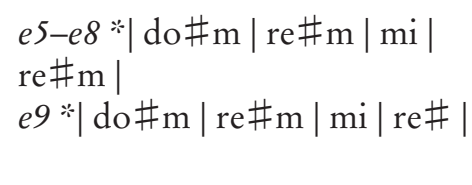 \\
\hline
\end{tabular}

Ediciones Universidad de Salamanca / 
Sobre el uso de los samples, se puede decir que están compuestos por estos bloques de acordes, por el punch que se emite en los subgraves y por un ritmo de tango con las siguientes figuraciones:

\begin{tabular}{|c|c|c|c|}
\hline \multicolumn{5}{|c|}{ Tabla 12. Samples rítmicos } \\
\hline Estribillo 1 y Estrofas & Estribillo 2 & Estribillo 3 \\
\hline 4 & 9 & 0 & 4
\end{tabular}

De esta forma, destaca la existencia de una oposición tímbrica entre elementos del flamenco y de las músicas urbanas que es el resultado mismo de la hibridación planteada. Y, como «[...] the timbre relates indexically to a musical style and genre, producing connotations of a particular culture or environment» (Tagg, 2013:306), es fundamental el empleo de la dicción andaluza, el ritmo de tango característico y las palmas para que el oyente relacione esta música con el flamenco. De igual manera sucede con el uso de ad-libs, efectos, sintetizador, samples e inclusión de subgraves para su vinculación con las músicas urbanas.

En la imagen, el videoclip empieza y termina con la misma idea: Rosalía tumbada en la cama en una determinada posición y vestida de blanco. Se trata de otra intertextualidad en la que se recrea el cuadro de Francisco de Goya (Fuentedetodos, 1746-Burdeos, 1828) titulado La maja vestida (1800-1808). Ello es un reflejo de la era postelevisiva en la que priman concepciones inter- $y$ transmediales -como la intertextualidad a través de una tradición o un cuadro en otro medio artístico-, la «interactividad e inmersión» -como la coreografía en el videoclipo «la identidad cultural»-representada por el Yeli, La maja vestida o el habla andaluza- (Auslander, 2008; Bolter y Grusin, 2000; Richardson et al., 2013: 20-31; Sedeño Valdellós et al., 2016: 334). El objetivo de parte de todos estos procedimientos no es otro que la creación de imaginarios en aras de ser relacionados con determinados géneros (Sedeño Valdellós et al., 2016: 337).

Por tanto, y como apunta Philip Tagg (2013: 238) sobre este tipo de procesos, el objeto de análisis adquiere las connotaciones que tiene el objeto referencial: $L a$ maja vestida. Estas serían la identidad española, puesto que es una obra conocida a nivel internacional, el majismo de la sociedad española de los siglos XVIII y XIX, muy relacionado con el flamenco, y un cierto exotismo. Es más, como el lienzo fue llamado «gitanas», durante un tiempo, entonces el significado de esta referencia incide en el factor intencional de atribuir a esta música un carácter u origen propiamente flamenco.

Todas las intertextualidades señaladas añaden diferentes niveles de comprensión, pero también provocan en el espectador una reacción y una reflexión, siempre y cuando se intente acceder al significado de dichos estratos. Así pues, junto con 
los elementos musicales ya analizados se teje una red llamada campo semiótico intertextual (López Cano, 2018). Es este proceso el que apela a la construcción identitaria la cual, según Simon Frith (1996: 111, 114), no sucede mediante la enumeración o inclusión de ciertos rasgos, sino que tiene lugar en la yuxtaposición de los mismos y la manera en que se interrelacionan: se trata de un proceso entre el individuo y la sociedad que se produce gracias a la experiencia previa, códigos, convenciones e ideologías.

Sobre la iconografía contenida en el videoclip se puede destacar el empleo del color blanco en los vestidos, que simboliza la deificación en tanto que se vincula con el luto blanco y el duelo regio o divino, y la inocencia, en yuxtaposición con el pelo negro de las bailarinas, reflejo de lo nefasto y advenimiento de la muerte. Así como las joyas de oro que refuerzan ese carácter de divinidad, poder y superioridad. Sin embargo, la presencia del rosa -en el neón circular, en los suelos, en el colchón y en los cojines de la cama- genera toda una estética basada en la sensualidad y la pasión (Chevalier y Gheerbrant, 1982: 671; Cirlot, 2004: 137, 344), lo cual entronca con el significado extraído de la letra. Pero lo más sorprendente es que durante el breve lapso entre [01:20-01:26] se detecta que la grabación se ha realizado en una iglesia. Algo que antepone lo ceremonial y sagrado a la pasión y deseo carnal ya aludidos.

A pesar de la supuesta continuidad del plano secuencia, el cual presenta un corte en [01:39] y su total fragmentación a partir del [02:18], lo visual marca una nueva división formal si se atiende al lugar donde suenan cada una de las partes de la canción y se acepta como transición el intervalo [1:39-1:54].

\begin{tabular}{|c|c|c|}
\hline \multicolumn{3}{|c|}{ Tabla 13. Estructura formal de la imagen } \\
\hline 1. ${ }^{a}$ SECCIÓN & 2. ${ }^{a}$ SeCCIÓN & 3. ${ }^{a}$ SeCCIÓN \\
\hline EstRIBILLO $1+E 1+E 2+E 3$ & ESTRIBILLO $2+E 4+E 5$ & ESTRIBILLO 3 \\
\hline$[0: \infty 0]$ & {$[0: 59]$} & {$[1: 54]$} \\
\hline
\end{tabular}

Es destacable la composición en el plano secuencia de los primeros planos de Rosalía en las partes que coinciden con las secciones de EstRIBILlos al producir una enfatización de la letra en dichos momentos (Sedeño Valdellós, 2016: 338). Otro aspecto reseñable sería la tercera sección. En ella se rompe el plano secuencia al realizar el giro en torno a la cama realizando catorce tomas entre [2:18-2:35]. El objetivo es acelerar el ritmo visual de la narración mediante ráfagas de tomas creando un punto climáctico en el videoclip que coincide con el final del mismo (Cook, 1999: 166). De hecho, esta sensación se intensifica debido a que se ubica durante el Estribillo 3, el cual introduce progresivamente el harmonizer, el jaleo y las voces en coro; se trata de un crescendo por adición. Los cortes, no obstante, 
se distribuyen tanto en los pulsos fuertes como los débiles causando, intencionalmente, una deformación del tiempo para potenciar la coreografía.

La parte coreográfica se integra por vueltas, braceos, movimientos de manos y caderas, típicos del baile flamenco, junto con varios pasos que se escinden de este género. En concreto, destacan dos eventos muy singulares. El primero sucede en la 2. ${ }^{a}$ Sección, durante [1:34-1:37], y consiste en un gesto con los brazos que imita los cuernos del toro. Es un elemento fundamental que engloba otra intertextualidad con la obra de Federico García Lorca (Granada, 1898-Granada, 1936). Se trata de la luna, la cual simboliza la muerte (Arango, 1995: 58) y se asimila al toro debido a la forma de los propios cuernos; adquiere un componente nefasto, mortuorio y de mal presagio a la vez que representa la fecundidad y lo sexual (Chevalier y Gheerbrant, 1982: 931). El segundo tiene lugar a comienzos de la 3. ${ }^{a}$ SECción remitiendo, y justificando, parte del título de la canción: «Cap. VIII: Éxtasis». Consiste en una serie de saltos, retorcimientos y gestos que evocan el acto sexual, pero que debido al montaje visual adquieren mayor fluidez. Además, el EsTRIBILlo 3 colabora a producir una atmósfera de intensidad narrativa con el crescendo por adición. Por último, Rosalía vuelve a adquirir la pose de La maja vestida para terminar el videoclip dando lugar a otro paso flamenco: el desplante.

\section{CONCLUSIONES}

El análisis efectuado muestra una parte del imaginario y la simbología que rodea el disco de El mal querer y, en concreto, del objeto de estudio. Específicamente, se distinguen la Luna, el toro, las joyas de oro, el color blanco y el negro para el vestuario y el pelo más el rosa para el atrezo y al resultado general de lo visual. A través de algunos de ellos se recurre a obras de arte referenciales en la historiografía pertenecientes a Lorca y Goya. También se alude a tradiciones propias de los gitanos que se enmarcan en el contexto del ritual y ceremonial junto con una presencia de la religión cristiana, casi imperceptible, que se manifiesta en la localización donde se ha grabado el videoclip y su decoración con dos cuadros de santos en las paredes. En este sentido, no se trata de proselitismo sino de ubicación en un marco contextual ligado a las tradiciones referidas y la religiosidad popular. Por todo ello, el objetivo principal contribuye a crear una estética determinada, en esencia híbrida, que genera un lenguaje propio, aunque entendible por la cultura hispana al estar profundamente arraigado en el imaginario colectivo. Además, todo este compendio del imaginario y lo simbólico añade capas de significados que originan procesos identitarios ya que son modos de diferenciarse y, a la vez, de integrarse en una subcultura urbana; en definitiva, se encaminan hacia la singularización de los fans. 
Como experiencia cinematográfica Rosalía plantea El mal querer como un potencial álbum visual que, si bien se trata de una estrategia de marketing, también pretende consolidarse como una obra de arte en sí misma. Es más, cada formato -el álbum, los directos o los videoclips- añade un tipo de información complementaria al resto de canales que reproducen esta obra. Cabe destacar que la misma producción constituye toda una red corporativista que ha sido la responsable de un absoluto éxito entre el público, especialmente en las vías de difusión que ha llegado a alcanzar. Reconocer la autoría de Antón Álvarez, El Guincho, Charm La'Donna o Henry Scholfield -por citar unos pocos- significa publicitar la nueva canción en la que han participado cada uno de ellos, lo cual permite introducir la canción en los sectores de audiencia de cada uno de estos colaboradores. Por otra parte, la diégesis del track analizado, la coreografía y la intención publicitaria de la música llevan a pensar que la categoría de videoclip sea mixta, lo cual se relaciona con una mayor posibilidad y voluntad de elaborar significados (Sedeño Valdellós, 2016: 337-338).

Desde el punto de vista de la semiótica, he procedido al estudio de ciertos tópicos en los géneros musicales hibridados: el flamenco, la electrónica y las músicas urbanas -estas dos últimas agrupadas bajo el término pop-. El objetivo de esta metodología ha sido profundizar en la significación a la vez que comprender cómo se han hibridado tales géneros. También he considerado oportuna la aplicación conceptual de clases de paradigmas, los procesos de indexicalidad y la identificación de procedimientos intertextuales puesto que añaden capas de significación que entroncan con las hibridaciones realizadas. Como señala Burke, «[...] musical bybridization may be analysed in terms of affinities or convergences. The attraction of the exotic often seems to lie in a distinctive combination of similarity and difference, rather than in difference alone» (Burke, 2009: 24). En virtud de ello, para cada elemento musical del flamenco he encontrado un rasgo yuxtapuesto que comparte estrechas similitudes, a saber:

1) Jaleo vs. ad-libs. En ambos contextos, en el flamenco y en las músicas urbanas como, por ejemplo, el rap, existe una notoria presencia de intervenciones espontáneas por parte del cantante, músicos o acompañantes que le rodean. Son convenciones que describen unos comportamientos vinculados a rituales propios de tales géneros.

2) Pronunciación andaluza vs. efectos (AutoTune y harmonizer). La equivalencia de la pronunciación en el flamenco con la música urbana y pop sería, aparte de la misma pronunciación, su edición mediante un software. Dicho de otra forma, sería la actualización de las voces flamencas a la era digital, aunque ya destaqué que tiene otro tipo de intencionalidad más en el epígrafe 3.2: la expresividad. Es el punto en común; es la convergencia. 
3) Ritmo de tango vs. subgraves. Ambos, por sus cualidades tímbricas, permiten al oyente identificar la música que suena con distintos géneros musicales. Concretamente con el flamenco y con la música electrónica e, inclusive, urbana. No obstante, el carácter rítmico de los subgraves suele trasladarse al plano flamenco evocando el sonido del taconeo -al menos, un taconeo muy conceptual- en el trabajo de Rosalía.

4) Cadencias andaluzas vs. samples. Quizá el hecho de que la cadencia andaluza aparezca hasta doce veces pueda parecer repetitivo ya que no es la única progresión existente en los acompañamientos flamencos. Sin embargo, esta idea adquiere una prolongación hacia el pop si se entienden esas cadencias como sampleos. Por otro lado, la gran diferencia con respecto de los géneros del pop -donde prima la tonalidad- es que esta secuencia se construye desde la armonía modal.

5) Cuartetas octosílabas vs. preestribillos y estribillos. Salvo excepciones, el flamenco no contempla el empleo de estribillos y sí recurre, con extrema frecuencia, a las cuartetas octosílabas; lo raro en el pop es encontrar esta métrica. En tal caso, son rasgos que divergen en este proceso de hibridación, pero que son necesarios y justificados en una canción con potencial promocional.

Acerca de las intertextualidades, los tangos de Málaga de la Repompa funcionan como justificación de su obra, e incluso como cantaora flamenca, mediante una cita a una artista bastante reconocida en el flamenco llamado clásico. La incorporación del Yeli sirve para construir un ambiente vinculado a lo gitano y, también, a lo tradicional y ceremonial dentro del marco narrativo del disco. $\mathrm{La}$ maja vestida remite al oyente internacional hacia su propio imaginario de lo que es el arte español y suscita una asociación de la música con la pintura como buenos productos debido a la consideración de obra de arte que ha adquirido el cuadro de Goya a lo largo de la historia. De manera análoga sucede con los símbolos del toro y la luna en relación con la obra de Lorca. Además, estas últimas tres referencias propician el desarrollo de procesos identitarios y un marco estético determinado. Por tanto, la red semiótica intertextual no solo permite acceder a las referencias empleadas, sino determinar sus connotaciones y sumar un nuevo elemento a dicho conjunto, aunque, ahora, con la reelaboración e interpretación de Rosalía y su equipo.

\section{BIBLIOGRAFÍA}

Arango, M. A. (1995). Símbolo y simbología en la obra de Federico García Lorca. Madrid: Fundamentos.

Auslander, P. (2008). Liveness: performance in a Mediatized culture. London: Routledge. 
Auty, S. y Lewis, C. (2004). The 'Delicious Paradox': Preconscious Processing of Product Placements by Children. En L. J. Shrum (Ed.), The Psychology of Entertainment Media: Blurring the Lines between Entertainment and Persuasion (pp. 117-133). Nueva Jersey: Lawrence Erlbaum Associates.

Bolter, J. D. y Grusin, R. (2000). Remediation: Understanding New Media. Cambridge: MIT Press.

Burke, P. (2009). Cultural Hybridity. Cambridge: Polity Press.

Chevalier, J. y Gheerbrant, A. (1982). Dictionnaire des symboles. Mythers, rêves, coutumes, gestes, forms, figures, couleurs, nombres. París: Éditions Robert Laffont, SA y Éditions Jupiter.

Cirlot, J. E. (2004). Diccionario de símbolos. España: Ediciones Siruela.

Cook, N. (1999). Analysing musical multimedia. Oxford: Clarendon Press.

Fabbri, F. (1981). A Theory of Musical Genres: Two Applications. En D. Horn y P. Tagg (Eds.), Popular Music Perspectives (pp. 52-81). Göteborg y Exeter: International Association for the Study of Popular Music.

Frith, S. (1996). Music and identity. En S. Hall y P. Du Gay (Eds.), Questions of cultural Identity (pp. 108-127). Londres: Sage Publications.

Gamella, J. F.; Fernández, C.; Nieto, M. y Adiego, I. X. (2012). La agonía de una lengua. Lo que queda del caló en el habla de los gitanos. Parte II. Un modelo de niveles de competencia y formas de aprendizaje. Voces y campos semánticos más conocidos. Gazeta de Antropología, 28(1), 1-28.

Giménez Rodríguez, F. J. (2008). Del Romanticismo pintoresco a la tendencia historiográfica: una aproximación al concepto de andalucismo en la música española. En F. J. Giménez Rodríguez, J. López González y C. Pérez Colodrero (Eds.), El patrimonio musical de Andalucía y sus relaciones con el contexto ibérico (pp. 153-168). Granada: Centro de documentación Musical de Andalucía-Editorial Universidad de Granada.

López Cano, R. (2018). Música dispersa. Apropiación, influencias, robos y remix en la era de la escucha digital. Barcelona: Musikeon Books.

Richardson, J.; Gorbman, C. y Vernallis, C. (Eds.) (2013). The Oxford Handbook of New Audiovisual Aesthetics. Nueva York: Oxford University Press.

Sedeño Valdellós, A.; Rodríguez López, J. y Roger Acuña, S. (2016). El videoclip postelevisivo actual. Propuesta metodológica y análisis estético. Revista Latina de Comunicación Social, 3(71), 332-348.

Tagg, P. (2013). Music's Meanings. A modern musicology for non-musos. Nueva York y Huddersfield: The Mass Media Music Scholars' Press, Inc.

Viñuela Suárez, E. (2013). El videoclip del siglo XXI: el consumo musical de la televisión a internet. Musiker, (20), 167-185. 


\section{WEBGRAFÍA Y DISCOGRAFÍA}

AA. VV. (2018). 19th Latin GRAMMY®Awards. The Latin Recording Arts \& Sciences, Inc. <Disponible en: https://www.latingrammy.com/es/nominees $>$ [Última consulta: 24 de febrero de 2019].

Caviar (2010-2019). Disponible en: <https://caviar.tv/portfolio\#music> [Última consulta: el 19 de marzo de 2019].

Gallardo, D. (2018). Rosalía: «Siempre voy a reivindicar a la mujer poderosa». Europa Press, 30/10/2018. Disponible en: <https://www.europapress.es/cultura/musica00129/noticia-rosalia-siempre-voy-reivindicar-mujer-poderosa-20181030181750. html> [Última consulta: 24 de febrero de 2019].

La’Donna, C. (1989-2019). Disponible en: <https://www.charmladonna.com/bio > [Última consulta: 20 de marzo de 2019].

Lipa, D. (2017). Dua Lipa - New Rules (Official Music Video). YouTube. Disponible en: $<$ https://www.youtube.com/watch?v=k2qgadSvNyU> [Última consulta: 19 de marzo de 2019].

Lipa, D. (1995-2019). Billboard. The hot 100. USA: Prometheus Global Media. Disponible en: <https://www.billboard.com/music/dua-lipa/chart-history/hot-100/ song/1028801> [Última consulta: 19 de marzo de 2019].

Timberlake, J. (1981-2019). The hot 100. USA: Prometheus Global Media. Disponible en: <https://www.billboard.com/music/justin-timberlake/chart-history/hot-100> [Última consulta: 24 de febrero de 2019].

Vila Tobella, R. (2018a). El mal querer [CD]. Sony Music (2017-2018).

Vila Tobella, R. (2018b). ROSALÍA - DI MI NOMBRE (Cap. 8: Éxtasis). YouTube. Disponible en: <https://www.youtube.com/watch?v=mUBMPajoL3o > [Última consulta: 24 de febrero de 2019].

Vila Tobella, R. (2018c). ROSALÍIA - BAGDAD (Cap. 7: Liturgia). YouTube. Disponible en: <https://www.youtube.com/watch?v=Q2WOIGyGzUQ> [Última consulta: 24 de febrero de 2019].

Vila Tobella, R. (2018d). ROSALÍA - MALAMENTE (Cap. 1: Augurio). YouTube. Disponible en: <https://www.youtube.com/watch?v=Rht7rBHuXW8> [Última consulta: 20 de marzo 2019].

Vila Tobella, R. (2018e). ROSALÍA - PIENSO EN TU MIRÁ(Cap. 3: Celos). YouTube. Disponible en: <https://www.youtube.com/watch?v=p_4coiRG_BI> [Última consulta: 20 de marzo de 2019]. 
